\title{
Geographic information systems (GIS) and remote sensing in aquatic botany: methodological aspects
}

\author{
R. Caloz ${ }^{\mathrm{a} * *}$, C. Collet ${ }^{\mathrm{b}}$ \\ a Department of Agricultural Engineering, Institute of Geomatic, Units of Spatial Information \\ System, Federal Institute of Technology, Lausanne, Switzerland \\ ${ }^{\mathrm{b}}$ Institute of Geography, University of Fribourg, Perolles, CH-1700 Fribourg, Switzerland
}

\begin{abstract}
A geographical information system (GIS) is much more than a computerized mapping system, it is a system designed to acquire, to manage and to process spatial information. In the current trend of a multidisciplinary approach of carth cnvironmental management, GIS offer a basis for integrating both data and analysis tools from a broad spectrum of scientific disciplines. This paper aims to present an understanding of concepts and methodologies involved in the field of GIS. It describes major steps involved in processing spatial information and illustrates the diversity among GIS uses. As a GIS is not only a set of computerized tools, but a fully integrated environment for the management and the analysis of spatial information, it requires from the applied fields of geosciences to interrogate about the nature and content of relevant information, as well as about the transfer of classical investigation and analysis methodologies. (c) 1997 Elsevier Science B.V.
\end{abstract}

Keywords: Geographical information systems; Spatial analysis; Remote sensing

\section{Introduction}

Geographic information systems (GIS) followed in the wake of the computer revolution of the last $20-30$ years. They are based on developments in computer

\footnotetext{
${ }^{*}$ Corresponding author. Tel.: +41216935782; fax: +41216935790; e-mail: regis.caloz@dgr.epfl.ch 
assisted drafting (CAD), which is the basis of digital cartography, as well as in statistical analysis and in databases.

To the non-specialist, GIS appears at first to be a simple tool for greatly facilitating the production of maps. All the data that they are based on are digitized; this process, however, has a logic that is not neutral and often constrains the user to modify the models and redefine the variables employed. Moreover, connecting graphic objects to a database adds to GIS a rich and powerful means of data storage and analysis.

To what extent can the biological sciences integrate these new methods into their procedures? More fundamentally, in what ways, once they are modified by developments in GIS, can one question the description and analysis procedures developed by aquatic botany specialists? An analysis of the articles published in this issue points out the most important elements. These become more clear after systematically examining the nature and logic of GIS. This question is not specific to aquatic botany, but can be put in similar terms for all the natural sciences.

Currently there exist many excellent basic texts on GIS, several of which are presented in the bibliography (Aronoff, 1989; Burrough, 1986; Bonham-Carter, 1994). In this article, we do not propose to present these in condensed form, but rather to establish some fundamental points to allow researchers in the natural sciences to grasp both the potency and, of course, the limits of this new approach and technique.

\section{The limits of digital cartography}

GIS is first seen as a means of computer assisted drafting (CAD), more generally as digital cartography. Drawing tables and ink have been replaced by computer software and high resolution printers. This view is too restrictive as it does not confer to GIS the potential gained through databases, the veritable heart of GIS. If graphic representation is clearly cartography's gravitational center, then that of GIS is its capacity to organize and process information in a structured system. Graphic representations become peripheral elements used to interrogate the database, formulate hypotheses or to communicate results of spatial analysis. A brief review of cartography's characteristics will make this more evident.

The map has long been the principal means of expressing and synthetically representing an analysis of a phenomenon taking place on the Earth's surface. Following the example of other disciplines, botany has effectively and frequently used this tool. The use of an evocative semiology endows the map with a considerable capacity to transmit the most pertinent results. It's limits, however, are a hindrance for many applications.

1. Though the map's synthetic nature offers the user dense and rich information, it is limited to only those messages that the authors wished to pass on. The primary data, as well as the results of intermediate analyses, are not present on the cartographic document. This situation does not allow the 
user, whose question deals with only a part of the synthesized parameters, to exploit them according to his or her needs.

2. For reasons of aesthetics and reading clarity, cartographic representationlimits the amount of information assigned to graphic units. Legends can be added, but only as a last resort, as the volume of information is limited and close scrutiny of the document is then necessary.

\section{Modeling geographic space}

Scientific method is based on a process of modeling reality. Since this is too complex to be grasped and comprehended in all its details, we create simpler representations. GIS is applied in disciplines whose study object is a portion of the terrestrial surface: a geographic space. (Brunet et al. (1992) define this concept as 'the terrestrial expanse used and managed by societies for their reproduction in the broad sense, that is, to procure food and shelter, but including all the complexity of social actions as well. It encompasses the totality of places and their relations. For this reason, it is at once a system of relations and an organized product resulting from the interactions between nature and societies'. More succinctly, the scope of geographic space is the study area, all the while including, where possible, the influence of its context.

Representations of geographic space correspond both to the perceived aims of the person(s) creating the model and to the characteristics of the natural phenomena present. The modeling process, however, is not independent of previous data processing stages. This is already true for conventional cartography in that graphic constraints orient the data synthesis process to a certain extent. For GIS, it is a question of putting the initial modeling into perspective with the constraints of computerization, that is, the reality model with the conversion of all the data describing the model into digital form. It is in this light that one must understand the stages of modeling geographic space proposed below.

What does one model in geographic space? In the first place, one identifies and represents the state of land use at a given moment, that is the presence and subsequent description of spatial objects or categories of natural or artificial objects (cities, ports, a coastal line, vegetation or soil structure considered to be homogenous, etc.). Secondly, one determines the state of a natural or social phenomenon, such as the meteorological situation (wind temperaturc, insolation), demography or population distribution. One models as well the change of a natural or social phenomenon, such as a migratory movement or the hydrological behavior of a watershed. These phenomena can concern the entire geographic space considered, or simply the behavior of a single spatial object, such as a river segment, a vegetation unit, etc. Lastly, one models the relations between objects or groups of spatial objects. These relations can be spatial, such as neighbourhood, or functional, such as the barrier effect of certain vegetation at a water's edge.

From a spatial point of view, this modeling process highlights two concepts: the spatial object (a limited space with a fixed geometry); and the spatial variable 
defined for each point of the geographic space. The spatial object is consistent with our usual understanding of reality, which consists of associating our observations to an abstract mental representation of the objects perceived, in the form of categories. The spatial variable, such as surface water temperature or soil texture, is defined for every point in space and is not limited to an object. The two concepts are in fact represented differently in GIS, as we will see later on.

Identifying objects or homogenous zones in geographic space consists of partitioning the space into spatial elements which geographers refer to as observation units. The observation unit is defined as a fundamental spatial element of geographic space. Its determination is relative, depending on the scale at which the phenomenon is studied, the study topic and the expert's subjectivity (Legros et al., 1996). A geologist does not identify the same observation units as the botanist or the land-use manager! A superior unit is not the sum of the units of which it is composed: a lake is not simply the sum of its biotic and abiotic components and therefore might have its own characteristics such as its overall physical and

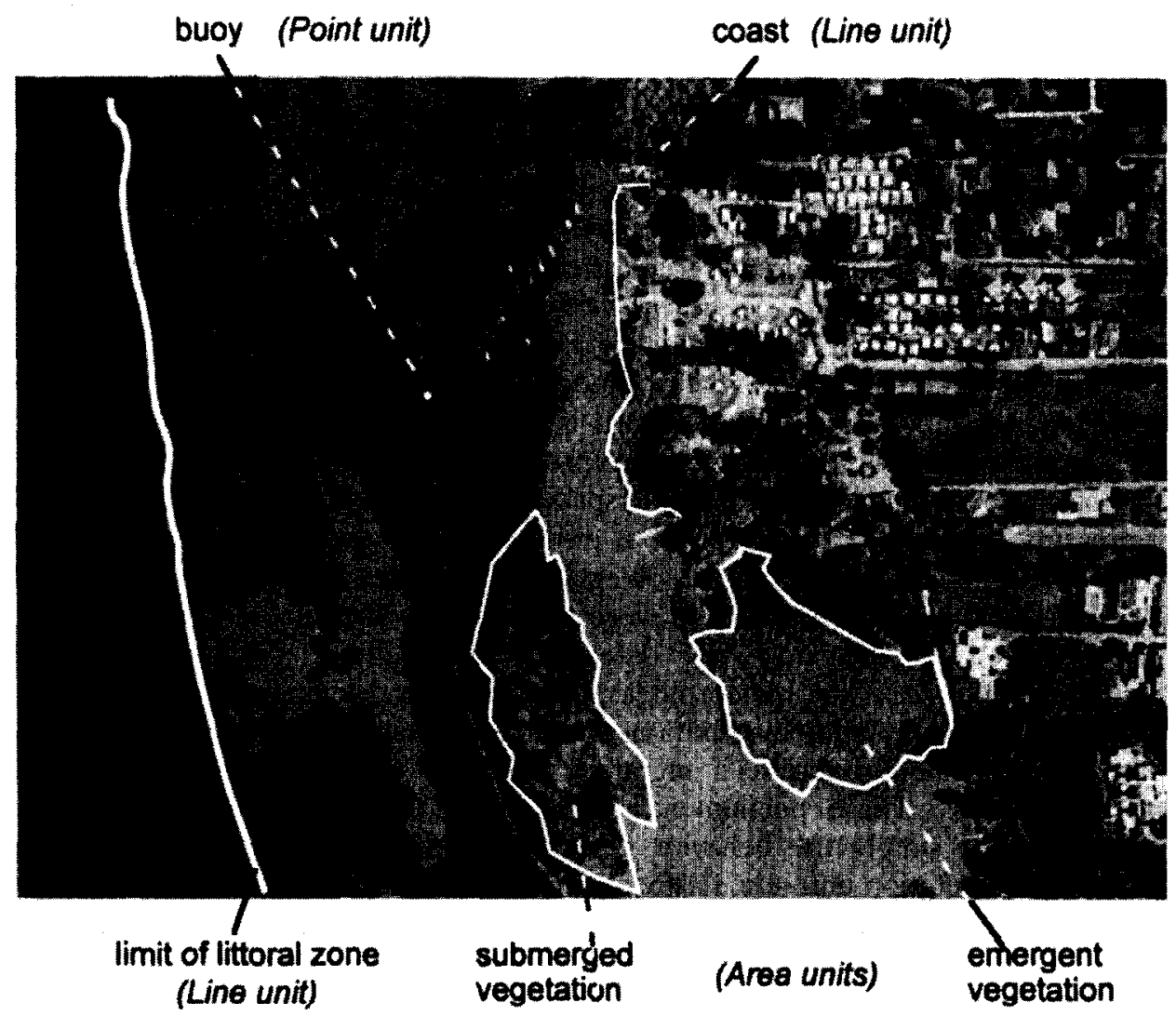

Fig. 1. Categories of observation units. 
chemical characteristics, its name and so on. This is therefore a relative notion that depends on the problem and the scale.

Moreover, an observation unit's existence can be concrete (a structure built for coastal protection) or abstract (a species' habitat zone). As soon as an observation unit is characterized by its attributes, its definition is reduced to the same. From a digital point of view, the attributes will be the only source for processing. Modeling spatial objects produces irregular observation units, as illustrated in Fig. 1.

For variables that are defined for all points in space (altitude, soil moisture, etc.) the situation is more complex. As it is hardly possible to express their value for every point in space since the volume of data becomes infinite, one chooses then to retain values only at certain regularly spaced points (at the intersections of a regular grid, for example), or to use an average value for the parameter within each cell of a regular grid.

The same approach can be used to describe land use. For each grid cell, for example, one assigns the type of land use occupying the majority of space in the cell (dominance rule). Fig. 2 presents this approach. In this case, the notion of object disappears.

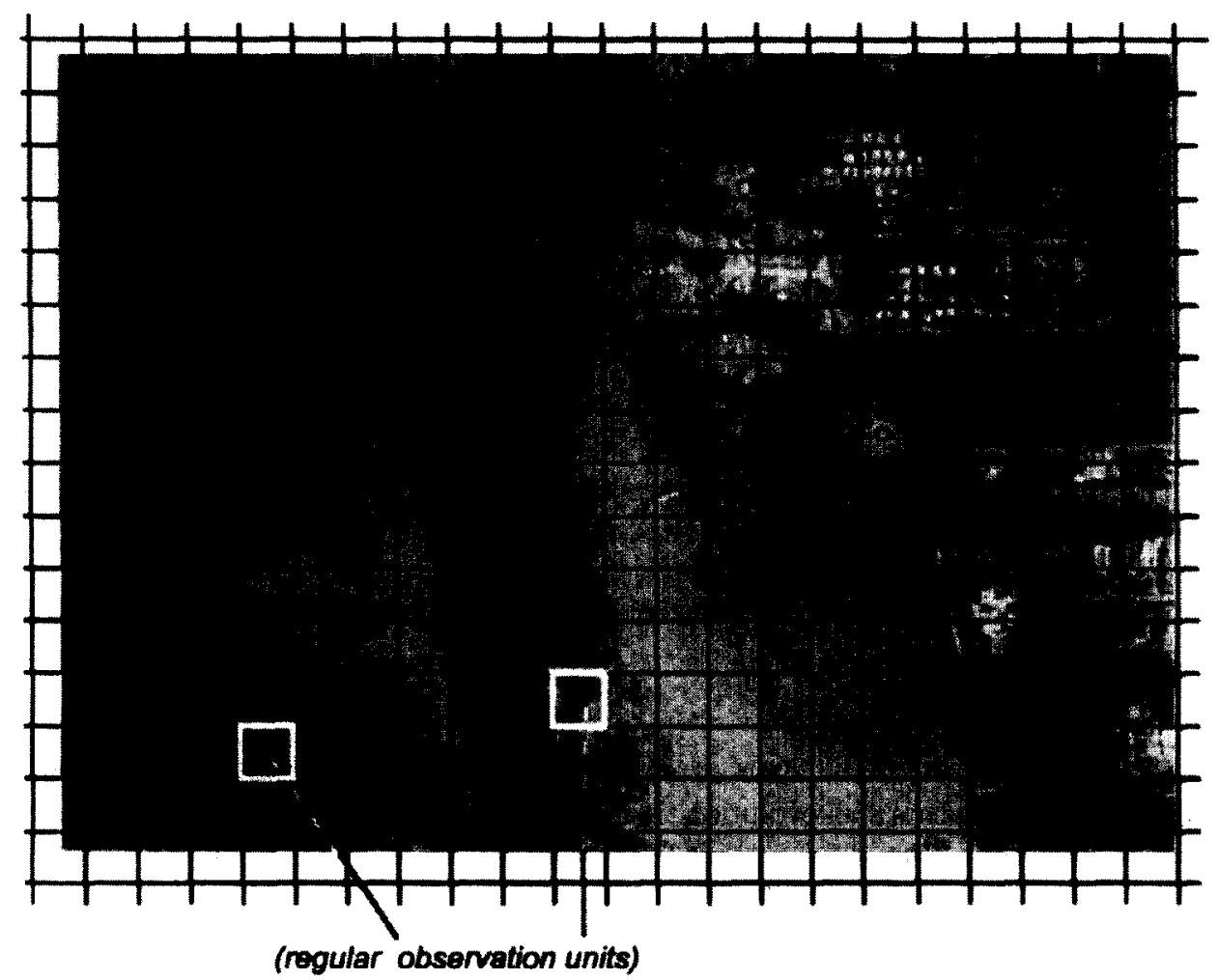

Fig. 2. Regular observation units. 
Other apportionments of space are possible, such as triangulated irregular networks (TIN). These represent an intermediate model between the two others. They are based on an irregular discretization of geographic space and are applied preferably to continuous variables. Its main advantage is that it allows the retention of measure points or structure lines, as in the case of surface topography (embankments). This is not possible with discretization based on a regular grid, in which case the values at the intersections of the grid are usually obtained by interpolating from irregularly distributed sample points. These are therefore estimated values, marred necessarily by imprecision.

The problem remains basically the same and we will not further develop other spatial discretization models here. We invite the reader to consult the abundant literature on this subject, in particular Burrough (1986), Bonham-Carter (1994) and Thériault (1994). The unit of observation is generally called spatial object or entity, the latter term more closely approaching computer database language.

\subsection{The dimensions of the observation unit}

Once geographic space has been modeled into regular or non-regular observation units, these are then characterized in terms of their dimensions: the spatial, thematic and temporal dimensions.

\subsubsection{The spatial dimension}

The spatial dimension takes location, shape, size and topology into account. Location follows the usual rules of georeferencing. Shape and size are a reflection of the geometric simplification possibly carried out on the object. Topology refers to neighbourhood relationships, i.e. what is next to what? Or in a network, what is attached to which segment? This notion satisfies a fundamental requirement of spatial analysis. For a representation in regular spatial units, the spatial dimension is reduced solely to the grid's resolution.

\subsubsection{The thematic dimension}

The thematic dimension refers to the object's pertinent characteristics according to the previously defined reality model. It specifically concerns the nature of the object, its physical, chemical, biological and social properties, as well as the functions linking it to other objects, for example the average flow rate of a river where it enters a lake.

\subsubsection{The temporal dimension}

This has to do with the date of an event associated with the object, or with information concerning its evolution. It is linked either to the object itself (date of data acquisition in the database), or to a parameter describing the object (average population of a city over the last 10 years). Incorporating the temporal dimension in a GIS presents some difficulties. It all depends on the speed of an object's or a variable's change. If the changes are slow, one must determine the update period for the information; in addition, one must decide whether to establish a history by 
retaining past versions of the object or by defining a synthetic attribute that describes the observed evolution. If the phenomenon evolves rapidly, the GIS is no longer adequate to describe it. Here it becomes necessary to turn to simulation models in which case the GIS is involved only to provide input parameters for the model or to accept the simulation results.

\subsection{Spatially referenced data}

Each observation unit's descriptive parameters, determined in light of the dimensions discussed above, are then spatially referenced data, also called an attribute in the context of its introduction into a database. This is to GIS what the atom is to matter: the basic element. Every analysis and every operation is carried out at this level.

\subsubsection{The georeference}

The spatial reference is a coordinate pair defined in a coordinate referencing system: for a point object, point coordinates; for linear or areal objects, the coordinates chosen to locate them (generally the gravitational center of the geometric unit).

\subsubsection{Nature and properties}

Spatially referenced data have different natures, specifically where their measure scale and their means of acquisition are concerned. These must be clearly identified in order to determine the appropriate logico-mathematical operations to apply when using the database. The measure scale can be nominal, ordinal or intervalratio. The Fig. 3 (Collet, 1992) synthesizes the properties of these three levels.

The means of acquisition as well as the extent of pre-processing of the data used in a GIS become extremely important when considering the precision or uncertainty affecting the data, as well as the uncertainty of data processing results. It is necessary as well to indicate whether the data were acquired directly for introduction into a GIS, or if they were digitized from an existing map. In the latter case, the modeling is not carried out for the objectives and constraints specific to GIS, therefore the data should be processed separately. In GIS modeling, these data fall into three categories: measured, interpreted and derived.

\begin{tabular}{|l|l|l|l|}
\cline { 2 - 4 } \multicolumn{1}{c|}{} & \multicolumn{1}{c|}{ Nominal } & \multicolumn{1}{c|}{ Ordinal } & \multicolumn{1}{c|}{ Interval-ratio } \\
\hline Information content & Identification & Identification, ranking & $\begin{array}{l}\text { Identification, ranking, } \\
\text { interval unit }\end{array}$ \\
\hline Permitted operations & $\begin{array}{l}=, \neq \\
\text { Some logical operators }\end{array}$ & $\begin{array}{l}<>=, \neq \\
\text { Logical operators }\end{array}$ & $\begin{array}{l}<,>,=, \neq,+,-,+, \\
\text { Logical and mathematical } \\
\text { operators }\end{array}$ \\
\hline Related statistics & mode, diversity & median, quantiles & $\begin{array}{l}\text { mean, variance, } \\
\text { correlation, ... }\end{array}$ \\
\cline { 2 - 4 } & \multicolumn{2}{|c|}{ Discrete (discontinuous) values } & Continuous values \\
\hline
\end{tabular}

Fig. 3. Properties of the three levels of measurement. 


\subsubsection{Measured data}

Measured data correspond in particular to the values of a variable obtained using a measuring instrument. Examples include pluviometry, digital satellite or aerial photography, water level at a particular location of a river, a soil sample's granulometry or a forestry site's inventory. Measured information is generally not the result of a calculation (other than those used for structural changes, unit transformation, etc.).

\subsubsection{Interpreted data}

Interpreted data, for example a landscape unit, is a synthetic, overall assessment produced by an expert. It is comprehended directly in the field or from an image and includes the knowledge and experience incorporated in the expert's synthetic view. It can not be produced automatically based on an inventory of criteria applied by the expert. However, this kind of data is partly intuitive, as much of the data is presented on thematic maps, such as pedological, geological or ecological maps. The expert proposes an apportionment of space that takes on a sense of its own, independent, to a certain degree, of the data that one assigns to these observation units. This is often true for pedological or ecological units, e.g. ecological maps of Quebec (Ducruc, 1991): surveys or field studies are carried out afterwards in order to characterize the previously defined spatial units so that a parameter can show strong spatial variability within a taxon, without calling the original partitioning into question. This type of data is marred with uncertainty due to the expert's inevitable subjectivity.

\subsubsection{Derived data}

Data is said to be derived when it is produced using separately acquired parameters and combined using logico-mathematical operations: slope and orientation calculated from a digital elevation model (DEM), soil potential for a particular species of plant. This category also includes data taken at sampling points and then estimated in other points of space through a regionalization process.

Data acquired by digitizing a cartographic document comes under a different logic. The map is the result of its own spatial modeling. If the information it contains is introduced into a GIS by digitizing graphic units and transferring symbolic data, the operator is required to estimate both the data's reliability and its precision and to enter this as so called meta-information into the database.

\section{Digital modeling}

The modeling of geographic space proposed here is intended to be general and as independent as possible of specific applications. It is related, however, to the digitization model made available through computer methods. Digitization is applicable for the two categories of data that describe observation units: the spatial dimension and the thematic attributes. A space is described in terms of an ensemble of geometric elements and their relationships. The thematic attributes are digitized in the form of a table in a database. 
The two forms of spatial partitioning proposed here produce two digital models of spatial reality: the vector or object model and the image model. Fig. 4 illustrates the two cases. The attributes are digitized differently in these two models.

\subsection{Object or vector model}

The name object is preferable to vector as it better reflects the nature of irregular observation units: entities of geographic space based on a mental abstraction.

The principal behind digitization in object mode is to associate one or several data tables to the graphic unit, as shown in Fig. 5.

\subsubsection{Digitizing the geometry}

This consists of digitizing lines, zones and neighbourhood relationships. Depending on the software's degree of development, this objective can be attained at two levels: non-topological and topological. The first takes all the data into account for graphic restitution of the units and connection with primary tables. It is based on a dictionary of the geometric units. The topological level uses the chain concept, in that a border between two zones is stored only once. Increasingly, the chain can

Modeled geographical space

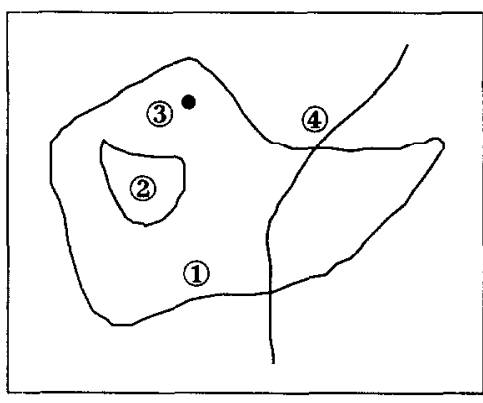

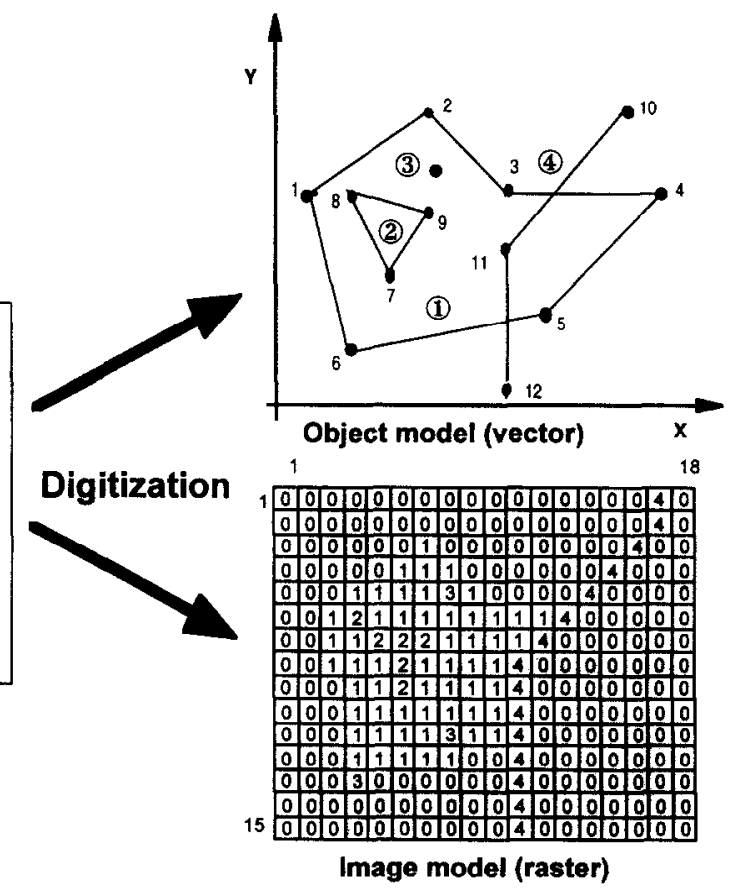

Fig. 4. Process of digitizing graphic units. 


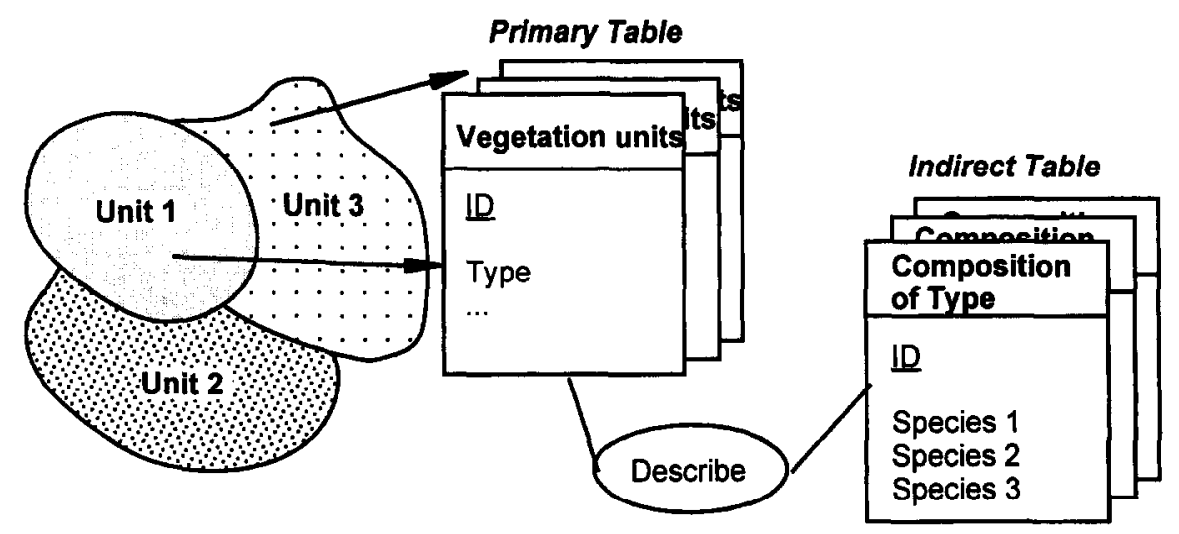

Fig. 5. Graphic unit connected to a primary and an indirect table.

also have an orientation, making it possible to define what is to the right or the left of it. Together these two features permit neighbourhood, inclusion or network analyses.

\subsubsection{Digitizing attributes}

The thematic attributes are digitized in the tables associated with the geometric objects (cf. Fig. 5). GIS programs offer the possibility to create direct and indirect tables independent from each other. Their connection is established through the language SQL (Structured Query Language) included in the functionalities of the software. If there is a large number of indirect tables requiring a more complex structure, one resorts to relational databases. The primary table, in this case, plays the role of access route to non-georeferenced data.

\subsection{Image model}

In the image model, the observation unit is the grid cell (pixel), which is defined by its resolution. The attribute is assigned to the pixel. There are as many grids as there are topics. The notion of object is entirely absent from this model. It is possible, however, to introduce a group of grid cells with the same attribute value in order to constitute regions and to create graphic units similar to the object notion.

\section{Precision-uncertainty}

Precision and uncertainty refer to the more general concept of data quality. In general the notion of precision is especially prevalent in measurements carried out in the exact sciences. It is defined as the random fluctuation observed during measurement that is expressed by the interval within which the exact value, which remains unknown, is located. For many natural phenomena this concept is too 
narrow and the notion of uncertainty is preferred. Uncertainty is the interval within which the variable measured is considered to be representative; a true value does not exist.

Precision and uncertainty are integral parts of the scientific development process. No step can be considered complete without clear, unambiguous information about previously acquired knowledge. What is the significance of these concepts for GIS?

Because georeferenced data are strongly anchored in official territorial data, the question of their quality has already been addressed in studies and directives. France, for example, has put together quality norms and criteria, called EDIGEO. The US has also written data quality recommendations, through the National Committee for Digital Cartographic Data Standards (NCDCDS) (Guptill and Morrison, 1995). The NCDCDS considers six aspects to determine quality:

- lineage (data history);

- positional accuracy;

- attribute accuracy;

- completeness;

- logical consistency;

- semantic accuracy.

For the purposes of this article, we would like to draw attention to some particular points concerning data acquisition and propagation of uncertainty during data processing.

\subsection{Data acquisition}

Spatially referenced data have been classed into three categories: measured, interpreted and derived. Each of these has its own particularities.

\subsubsection{Measured}

Depending on the measure scale, precision and uncertainty are estimated based on the means of acquisition and statistical tools.

\subsubsection{Derived}

The derivation process propagates any uncertainty found in each of the primary parameters. Threc situations will be cxamined later on. Among the types of derived data, one class of variables occupies a significant position in GIS applied to the natural sciences: regionalized variables, as defined by Matheron (1971) and Krige. These are variables defined for every point in space and assumed to present a spatial autocorrelation. They are acquired by sampling and then estimated by interpolation at representative points in the data system. The law describing how a spatial variable evolves as a function of its distance from a measure point is examined using statistical instruments of structural analysis (variogram). The advantage of Matheron's approach lies in the possibility to estimate the uncertainty affecting the interpolated values. 


\subsubsection{Interpreted}

This type of data is synthesized, that is it includes parameters measured by sampling within the spatial unit together with an assessment by the expert. The corresponding observation units are abstract (see Section 3). Besides the measurement precision of each of the parameters, the data also incorporate some subjectivity that is difficult to control. For the same topic, another expert can propose a different spatial apportionment. This subjectivity, recognized and acknowledged in cartography, has been translated until now by the fact that several disciplines' maps are signed by the author (geology, pedology, etc.). Computer system use is not well adapted to this situation. In fact, two types of information are merged during this process: the perception of the expert who determines the bounds of the observation units and the sampling measurements taken within the observation unit. In our opinion, it is preferable to separate these two types of data acquisition and maintain the data separately. From this point of view, it seems equally necessary to reexamine the sampling schema in order to render it independent of the initial partitioning. Interpreted measurements also encounter semantic difficulties between those who produce the data and those who use them.

\subsubsection{Propagation}

The main difficulty in estimating the uncertainty of a result from a spatial analysis procedure stems from the fact that one often processes and simultaneously combines data from the three measure scales. In fact, we can be confronted with the three following situations:

1. The processing brings into interaction only data of interval-ratio measure scale or variables presenting fluctuations that can be adjusted using a probability distribution law. At this level, there is a rich panoply of statistical tools available to quantify the uncertainty affecting the final result.

2. The variables' fluctuations can not be adjusted by a probability distribution. In this case, several authors suggest resorting to fuzzy logic, which proposes a possible empirical distribution. At this level as well, it appears possible to propose an interval of uncertainty for the processing results.

3. Data of the three measurement scales have been grouped and combined. The corresponding logical operations (cf. Fig. 3) do not allow a quantitative transfer between the different levels. It is up to the responsible operator to estimate the affects on the final result and to formulate the range of validity in terms of meta-information.

\section{Utilizing the geographic database}

Exploiting the geographic database (GDB) satisfies the needs of several complementary end uses, which themselves have implications for the reality model and therefore the nature of the GDB, as we have seen. The utilization procedures are 


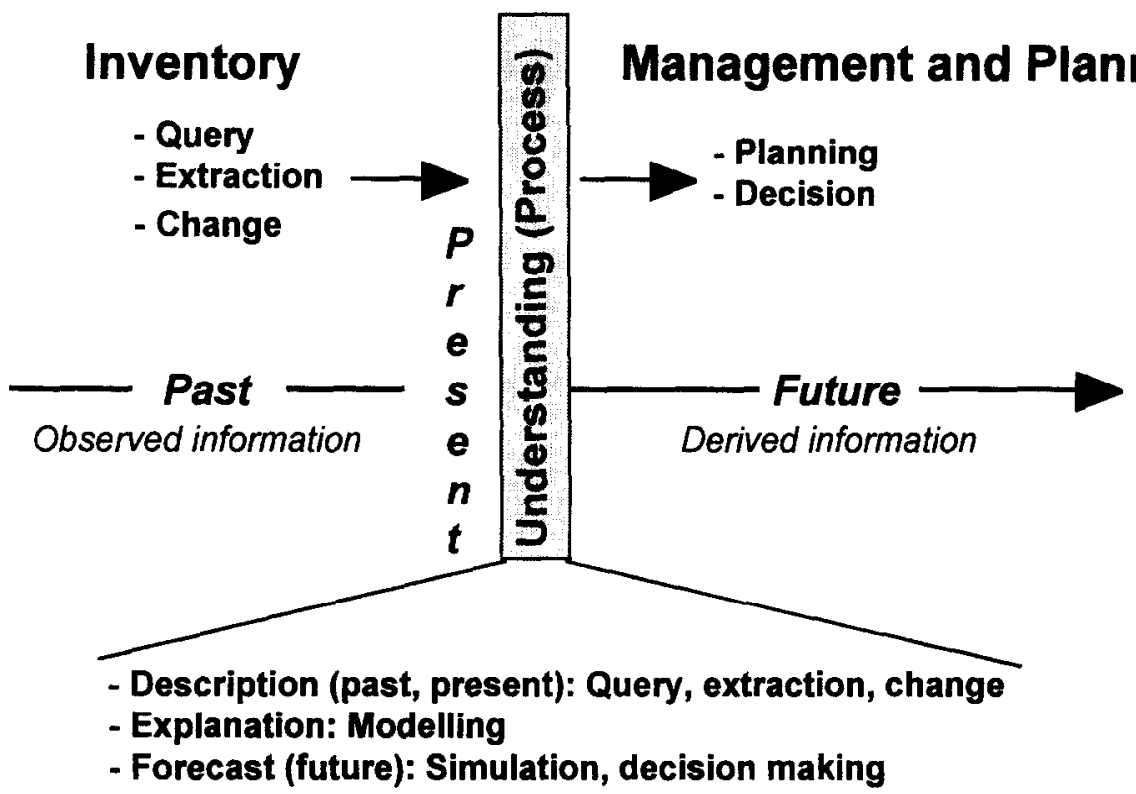

Fig. 6. Contexts for exploiting the geographic database in a GIS.

tightly linked to the tasks to be accomplished using the GIS. They can be organized into three categories (Fig. 6): spatial inventory, spatial analysis and spatial management.

\subsection{Creating a spatial inventory}

This is a basic approach permitting one to take account of the nature, the characteristics and the diversity of the objects which constitute the space. It is the descriptive and diagnostic phase for the objects in the study region and answers, above all, questions of object content and location. In the temporal dimension, it deals with information - observation results - on the past and the present of the reality considered. This inventory of spatial objects orients the procedure and thus the representation of reality, toward an object type model. One can see that in terms of software, GIS in object structure most effectively accomplishes the tasks of spatial inventory. There are two, often complementary, types of tasks: cartographic representation on one hand and thematic and spatial query on the other.

\subsubsection{Thematic and spatial query}

This query procedure selects and defines objects contained in the different layers that satisfy thematic or spatial criteria established by the user. A request language has been developed for non-spatial databases that allows object selection based on thematic criteria: SQL (Structured Query Language), based on logical operators. In 
order to include spatial criteria in the requests, SQL has been enriched with topological spatial operators. Subsequently called GQL (Geographical Query Language), it can be found in most GIS programs with object structure. These thematic and spatial queries produce a subset of selected objects and their attributes, organized in table form.

\subsubsection{Cartographic representation}

This is a powerful means of graphically expressing objects' thematic and spatial characteristics simultaneously. Visual perception gives an overall view of the objects' position, form and arrangement, as well as their thematic content being considered. The map provides the reader with information at two scales: a local scale detailing each of the objects in its two dimensions (spatial and thematic) and a regional scale presenting the spatial arrangement of their attributes. This type of cartography, with synthesis as its goal, long precedes computer technology. The result of this graphic expression is a synthetic map, also called an interpreted map, produced using an analog support such as paper, or a digital support transcribed graphically onto a computer monitor.

Cartography in GIS goes beyond the graphic expression of inventory or selection results, in that it also serves as a highly effective interface for spatial interrogation. A different type of cartography than the synthetic map, in this case it is only a reflection of the GDB elements and can thus be called a descriptive map. Selecting one or several objects on the monitor serves as an interrogation key for their attributes as well as their neighbourhood. In the context of GIS, therefore, cartography is not only a means of expressing results graphically, but also an interactive query language, complementary to GQL.

\subsection{Analysing space}

Parallel to studying the state and evolution of the characteristics of objects in a space, spatial analysis permits one to describe and model these phenomena's distribution in that space. This concerns the spatial dimension's properties such as geometry, topology, arrangement, proximity, accessibility and dynamics (Fig. 7).

It thus offers the possibility to comprehend the phenomena's spatial and dynamic interactions. This analysis approach originates from a view of reality that is complementary to that of object inventory. Although both reality models offer spatial analysis capabilities, the object model is particularly suited for dealing with flow processes in networks. For other types of spatial analysis, the image model is preferable. In this context, the spatial objects are actually the product of the phenomena's spatial distribution and interactions. For example, one can consider the presence and extent of a vegetation zone to be the result of the characteristics and interactions of phenomena, both physical and human. Analysing the entities that compose this zone will produce the zone's bounds, according to the reality modeling process presented at the beginning of this article. In order to study the spatial distribution and interactions of the phenomena in the zone, it will be necessary to present reality as an ensemble of spatial entities which are arbitrary 


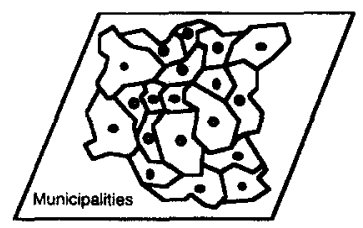

Geometry

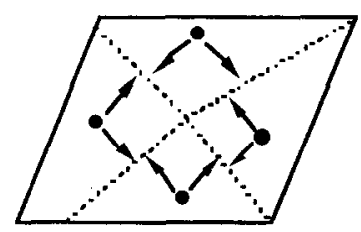

Proximity

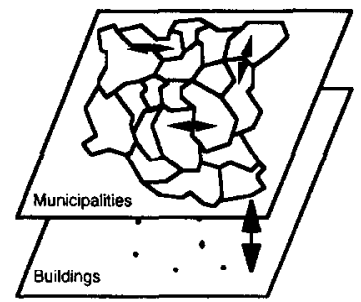

Topology

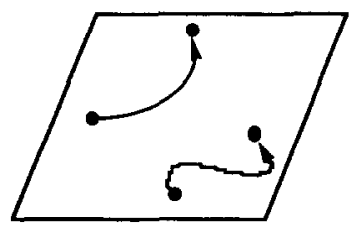

Accessibility

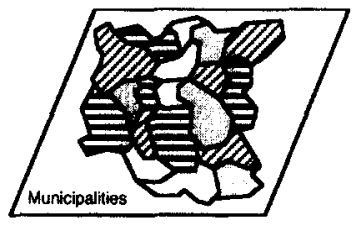

Pattern

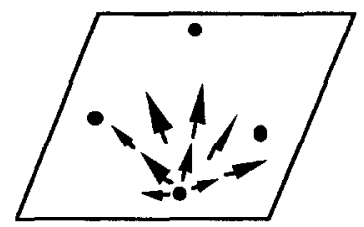

Motion

Fig. 7. Different properties of the spatial dimension.

and regular in shape and smaller in size than the objects to be produced. The image model proposes just such a vision of reality, in which each cell (pixel) of the grid contains a characteristic for each of the phenomena considered.

Two levels of spatial analysis can be identified: describing the spatial distribution of the phenomena's characteristics and modeling their interactions (spatial processes). In order to accomplish these tasks, GIS in image structure proposes a complement to statistical tools: analysis tools based on logico-mathematical operators, allowing the simultaneous consideration of spatial, thematic and temporal dimensions. This type of approach allows one to model, for example, reforestation linked to agricultural decline, or to simulate the influence of vegetation on littoral erosion. In the study of spatial processes, space can be modeled at different levels of complexity, from a flat isotropic plane to a skewed anisotropic volume (Fig. 8).

\subsection{Managing space}

Spatial management requires knowledge of the organization and history of the objects in the space, comprehension of the processes acting on them and a planning project. We note here that data on the past and present reality and an understanding of its functioning are necessary to design development scenarios for the future. We have seen that GIS provide a favorable environment for realizing these first two stages of spatial management. Let's now look at GIS' contributions to the establishment of a development strategy. One of management's fundamental components is the project, a concept outside the domain of scientific investigation, 

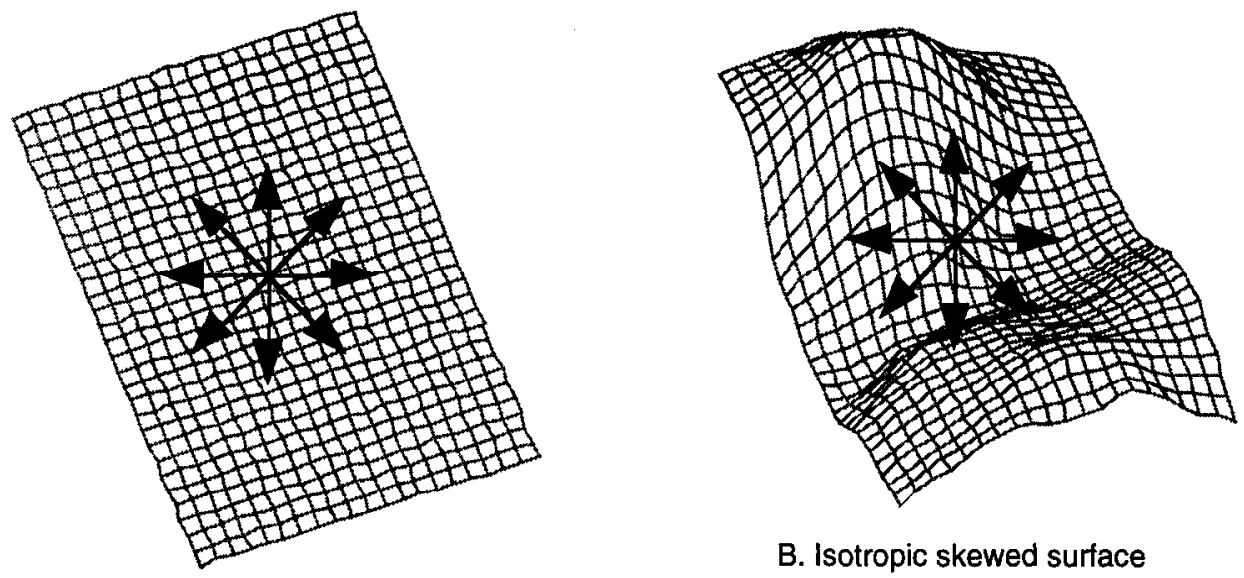

\section{B. Isotropic skewed surface}

A. Isotropic plane surface
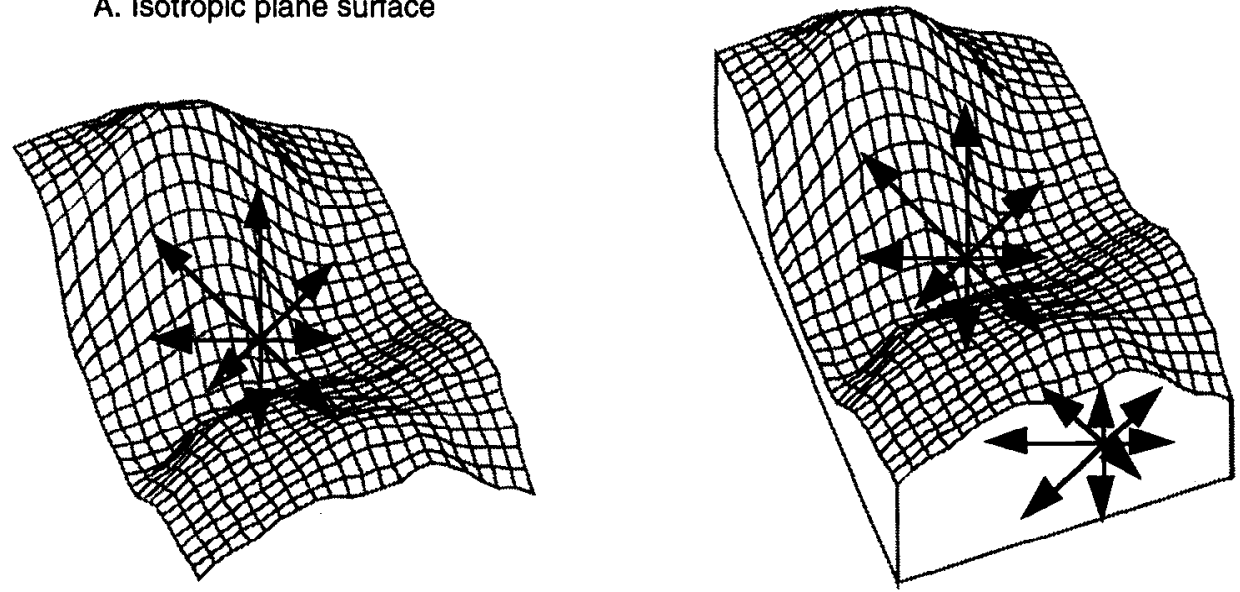

C. Anisotropic skewed surface

D. Anisotropic skewed volume

Fig. 8. Different levels of complexity of modeled space.

having more to do with the political and social spheres. Nevertheless, once this development project has been defined for a certain space, GIS can contribute in three ways: defining development potentials in this space, evaluating different scenarios and decision making.

The study of development potential presents the aptitude of each spatial element being studied for satisfying a particular land use (particular development goal). It draws not only on the thematic characteristics of the elements, but also on their neighbourhood and their spatial arrangement.

Evaluating development scenarios calls on procedures for simulating spatial dynamics. It proposes answers to the question 'what would happen if...?'.

Decision making regarding land management and development questions is a 
complex and iterative process involving actors from different political, social, economic and scientific backgrounds. GIS offers this situation a multidisciplinary environment of dialogue, evaluation and decision making aid.

\section{Remote sensing}

Remote sensing is above all a technique based on measuring the electromagnetic energy reflected or emitted by the surface of geographic space. The product is either a photograph reproduced on a graphic support (paper, film, monitor), or a digital image having the same properties as the image model described above.

The information in the image is spectral. Only through a visual or digital interpretation process can one attribute thematic significance to the pixels or groups of pixels (classification).

Along with its spectral properties, an image is characterized by its spatial resolution. This is the limiting factor in determining the scale at which one can exploit the data extracted from the image.

Aerial remote sensing principally consists of obtaining photographs with resolutions ranging from some tens of centimeters to several meters. Scanning radiometers are also utilized, although they are more in the domain of satellite remote sensing. The market is as yet dominated mostly by civilian programs based on producing high resolution multispectral images; a new generation, however, of very high resolution radiometers $(<10 \mathrm{~m})$ has been put in orbit and is already producing images with resolutions of 5-8 $\mathrm{m}$. Examples include the following missions: IRS-1C (5-8 m), India, 1996; Early Bird (3 m), USA, 1996; SPOT 5 (5 m), France, 2000.

\subsection{Exploiting the images}

Aerial photography and, to a lesser extent, satellite photography have important applications in photo interpretation. This procedure involves the determination and identification of observation units in addition to the determination of thematic attributes. As with any image, the resolution determines the application scale.

One application that has long been restricted to aerial photography has been extended to satellite images: photogrammetry. Pairs of SPOT satellite images taken under good atmospheric conditions and within a short time interval allow a planar precision of approx. $10 \mathrm{~m}$ and a vertical precision of approx. $20 \mathrm{~m}$.

For aquatic habitats, multispectral images with a band in the blue range (0.4-0.5 $\mu \mathrm{m})$ are preferable. Water reflects these wavelengths 1-10 times more strongly than those in the red, depending on water quality (Davis et al., 1978). Spectral variations at the water surface are then visible in the image, preparing the way for interpretation. At the same time, the blue penetrates the water to a greater depth than the other wavelengths. In shallow depths, the reflection from the bottom is still sufficient to detect the presence of vegetation classes. As with several remote sensing applications, this procedure is employed alongside along with in situ observations, thus facilitating the latter's regionalization. 


\subsection{Digital image analysis}

Satellite remote sensing was developed mainly around digital analysis of images. The processing can be grouped into four categories: radiometric and geometric corrections and transformations, image enhancements, production of thematic indices (biomass, etc.) and creation of zones in the image by pixel classification. The first category is a prerequisite to exploiting the image and the second modifies the image's visual aspects to make interpretation easier. These operations are not examined in this article; we will point out, instead, just a few of the classification procedures' particularities.

\subsection{Creation of zones in the image using segmentation and classification}

Zoning the image corresponds to the process of modeling geographic space. It permits one to extract spatial features. Several ways are possible, as summarized in Fig. 9.

The pixel approach groups pixels into spatial objects only on the basis of their spectral likeness. The two other approaches integrate both the thematic (spectral) and spatial dimensions. As the contextual approach examines each pixel within its neighbourhood (window), the feature approach identifies first spatial features through morphological analysis of the image.

\subsection{Zoning the image into vegetation classes}

The major difficulty stems from the frequent heterogeneity of vegetation units. According to criteria based on dominant occupation or on a particular interest in a category of plants, the botanist assigns a vegetation class to a zone. Often one

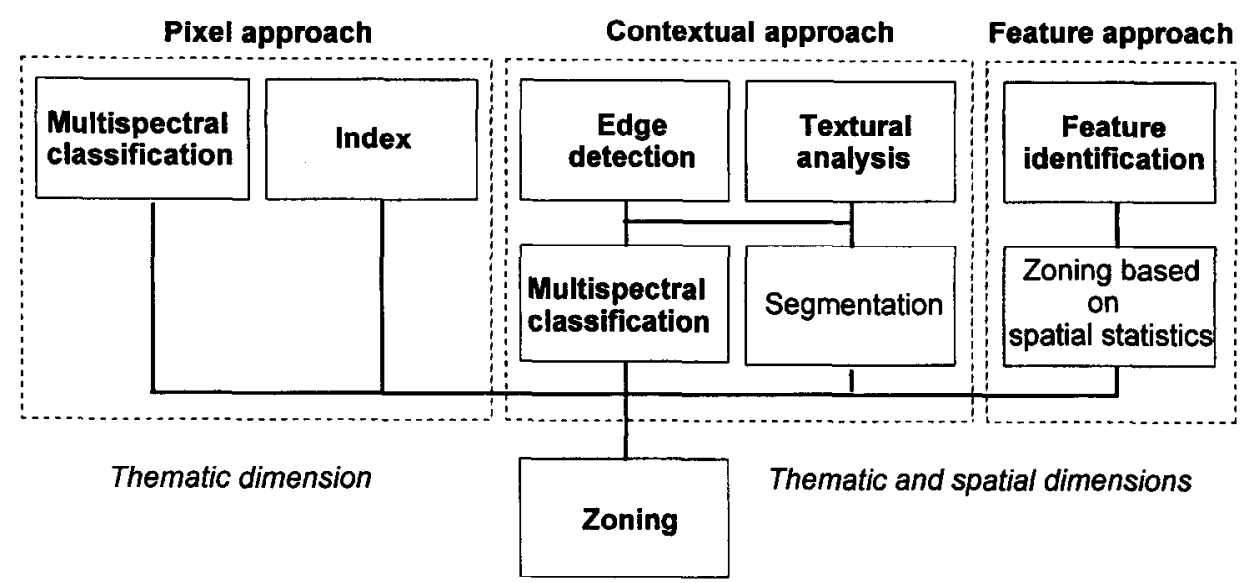

Fig. 9. Different approaches for segmenting an image into thematic zones. 
produces a spectral signature that is not sufficiently distinct from one produced for another habitat. A classification process applied to the image will not produce zoning that is easy to interpret thematically. The limits of the zones will not be clear, appearing somewhat like lace, or having areas 'moth-eaten' by pixels assigned to other classes. This situation is unacceptable cartographically, as well as for the geometric partitioning of observation units.

Because georeferencing is a primary aspect of GIS documents, the image and the products of the image must be processed to achieve cartographic quality, that is, rectified geometrically so that they conform to cartographic projections. If a digital elevation model is available, it is advisable to create an orthographic image as a background on the monitor; this can provide the user with rich and detailed information for putting spatial units into context. At this point, aerial and satellite photography guarantee continuity between the large and medium scale.

\section{Conclusions}

Compared to traditional and digital cartography, GIS contributes a new dimension to the modeling of geographic space: from a geometric point of view, through object and image models and, from a thematic point of view, through the connection to relational or object oriented databases.

Due to the richness of the data from remote sensing images, GIS represents an effective environment for the maintenance and exploitation of data describing our overall environment. Used in conjunction with simulation models for rapidly evolving phenomena, they provide the means for developing scenarios, contributing to sound decision making and finally, contributing to a better management of our biosphere.

The logic behind the digitizing process that governs the use of GIS is not neutral for any of the disciplines that use it. It necessitates, in many disciplines including botany, a revision of conventional approaches. It is up to each of us to judge whether this new approach offers the means of investigation and comprehension that can lead us to new advances in the state of our knowledge.

\section{References}

Aronoff, S., 1989. Gengraphic Information Systems: A Management Perspective. WDL Publications, Ottawa, p. 294.

Bonham-Carter, G.F., 1994. Geographic information systems for geoscientists: modelling with GIS. In: Computer Methods in the Geosciences. Pergamon, Ottawa, p. 398.

Brunet, R., Ferras, R., Thery, H., 1992. Les mots de la géographie - dictionnaire critique. In: Collection Dynamiques du Territoire. RECLUS, Montpellier, Paris.

Burrough, P. A., 1986. Principles of geographical information systems for land resources assessment. In: Monographs on Soil and Resources Survey 12. Clarendon Press, Oxford, p. 194.

Collet, C., 1992, Systèmes d'information géographique en mode image. In: Collection Gérer l'Environnement. Presses Polytechniques et Universitaires Romandes, Lausanne, p. 186.

Davis, S.M., Landgrebe, D.A., Phillips, T.L., Swain, P.H., Hoffer, R.M., 1978. In: Swain P.H., Davis, S.M. (Eds.), Remote Sensing: The Quantitative Approach. McGraw Hill, New York, p. 396. 
Ducruc, J.P., 1991. La Carte Écologique: Son Contenu et ses Utilisations. Ministère de l'Environnement du Québec, Québec.

Guptill, C.S., Morrison, J.L., 1995. Elements of Spatial Data Quality, Pergamon, Oxford, p. 202.

Legros, J.P., Kölbl, O., Falipou, P., 1996. Délimitation d'unités de paysage sur des photographies aériennes, éléments de réflexion pour la définition d'une méthode de tracé. Etud. Gestion Sols 3 , 113-124,

Matheron, G., 1971. The theory of regionalized variables and its applications. Les Cahiers du Centre de Morphologie Mathématique de Fontainebleau, Ecole Nationale Supérieure des Mines de Paris.

Thériault, M., 1994. Systèmes d'information géographique - concepts fondamentaux, LATIG. Dép. de Géographie, Univ. de Laval, Québec, p. 165. 